\title{
Eosinophils and cancer - mini review
}

Keywords: eosinophils, white blood cells, adaptive immunity, anti-cancer immunity, eosinophils, tumor cells, cytokines; cancer

\section{Mini review}

Eosinophils are a type of granulated white blood cells produced in the bone marrow that makes up about 1-5 percent of the total number of white blood cells. These granulated cells are generated by the process of hematopoiesis in the bone marrow and then migrate into the blood stream and extrasavate to tissue sites. ${ }^{1}$ They circulate at low levels in the blood in healthy individuals but marginate to tissues, where they are implicated in the regulation of innate and adaptive immunity. During normal homeostasis, eosinophils can be detected outside blood vessels in organs such as lower GI tract, ovary, uterus spleen and lymph nodes but not in the lung skin or esophagus. As a key player of the immune system eosinophils play an important role in combating parasitic infections in vertebrates and potentially cancer cells. Eosinophils can also play a pro-inflammatory role and particapate in the pathogenesis of allergic disorders including asthma. Eosinophils secreted chemokines and other cellular mediators participate in immunomodulation and tissue remodeling.

As multifunctional leukocytes, eosinophils have also been indicated in anti-cancer immunity. Published studies have suggested an association between allergic conditions and a trend of decreased risk in numerous malignances. Moreover, eosinophil infiltration in tumor tissue is considered an independent prognostic factor. Eosinophils are often recruited to tumor sites, where eosinophil granule proteins and cytokines are released upon activation, which in turn damage and kill tumor cells. On the one hand eosinophil-mediated organ damage has been of concern ${ }^{5}$ eosinophil infiltration in tumor tissue is considered an independent prognostic factor. Eosinophils are often recruited to tumor sites, where eosinophil granule proteins and cytokines are released upon activation, which in turn damage and kill tumor cells. In the last decade, a number of patents based on potential cancer therapy using eosinophilic cytokines have been awarded. ${ }^{6}$ Highlighting the promising targeted therapies with eosinophilic cytokines as a novel perspective to combat cancer. ${ }^{7}$ More recently it has become apparent that immune system cells and cytokines play essential roles in both mammary gland development as well as breast cancer. ${ }^{7-10} \mathrm{~A}$ study by Peter MS et al indicated that few disorders specifically affect breast skin, but the nipple-areola complex should be approached with a different set of diagnostic considerations. ${ }^{11}$ While another study by Andersen et al showed Eosinophil counts associate with hematological malignancies and mortality even below the definition of eosinophilia. They deduced that physicians encountering patients with mild-tomoderate eosinophilia according to traditional definitions confered maximally increased risks of subsequent/subclinical hematological malignancy. ${ }^{12}$

Although earlier studies in mice suggested that eosinophilderived IL-4 was required for mounting a Th2 response during immune reactions to intraperitoneally injected Schistosoma mansoni eggs, ${ }^{13}$ more recent studies have shown that ablation of eosinophils
Volume 4 Issue 4 - 2016

Terlika Pandit Sood

Clinical Research Coordinator, Perlmutter Cancer Center (Radiology), USA

Correspondence: Terlika Pandit Sood, Clinical Research Coordinator, Perlmutter Cancer Center (Radiology), Room 0234, 160 East 34th Street, New York, NY 10016, USA, , Tel |-2|2-73|-53|3, Email Terlika.sood@nyumc.org

Received: October 29, 2016 | Published: November 25, 2016

from mice had negligible outcomes, or even inhibitory effects, on helminth larval or egg expulsion in $\triangle \mathrm{dbl-GATA}$ or PHIL mice. ${ }^{13}$ These experimental findings imply that eosinophil-derived cytokines, chemokines, and growth factors have no specific role in the regulation of parasitic worm diseases, in contrast to the classically held notion that eosinophils are important in parasitic worm expulsion. Karagiannis et al., ${ }^{14}$ hypothesized that the unique properties of IgE, a class of tissue-resident antibodies commonly associated with allergies, which can trigger powerful immune responses through strong affinity for their particular receptors on effector cells, could be employed for passive immunotherapy of solid tumors such as ovarian and breast carcinomas. A possible interaction between macrophages and eosinophils in ductal morphogenesis is considered, along with the roles of other chemokines. This role of macrophages in normal development also appears to be subverted by tumors of the mammary gland to promote the escape of the tumor cells from the local environment and enhance their rate of metastasis. These data emphasize the dual role of macrophages in the promotion of epithelial growth in normal and cancer states ${ }^{15}$ Eosinophils are correlated to linked to a favourable prognosis in colorectal, breast and prostate cancers. $^{16}$

In other studies, certain types of lymphoid and solid tumors have been associated with the infiltration of eosinophils into cancerous tissues, ${ }^{16-18}$ particularly specific lymphomas and Hodgkin's disease. Although tumor-related eosinophilia was considered to be an epiphenomenon arising from the spontaneous elaboration of IL-5 from tumor cells, or overproduction of $\mathrm{T}$ cells during chemotherapy with IL-2, ${ }^{19}$ there is evidence of eosinophil activation as a result of IL-2 anticancer therapy. ${ }^{20,21}$ In some cases, tissue eosinophilia is considered to be a positive prognosis for head and neck cancers ${ }^{22}$ and advanced bladder cancer. ${ }^{23}$ Specifically, in oral squamous cancer, eosinophils 
are a positive prognosis for early stages of disease (Stages II and III), but an unfavorable prognosis for advanced cases (Stages III-IV). ${ }^{24,25}$

The involvement of eosinophil-derived cytokines, chemokines, and growth factors in neoplasias associated with eosinophilic infiltration is partially understood. In oral squamous cell carcinoma with tumor-associated tissue eosinophilia (TATE), the source of CCL11/ eotaxin is apparently eosinophils. ${ }^{26}$ The cultured oral squamous cell carcinoma cell line SCC9 secretes chemotactic prostaglandin D2, which promotes eosinophil transmigration, thus providing a signal to recruit eosinophils into tumor masses ${ }^{27}$ Eosinophils that infiltrate into tumors/lymphomas also express IL-6, TGF $\beta$, and CCL24/eotaxin- $2 .{ }^{28}$ On-going efficacy, safety evaluations and future first-in-man clinical studies of $\operatorname{IgE}$ therapeutics constitute key metrics for this concept, providing new scope for antibody immunotherapies for solid tumors.

However, while numerous studies have highlighted a role for eosinophil recruitment and activation in many types of cancers, and that eosinophils express receptors and mediators shared with cytotoxic $\mathrm{T}$ cell, ${ }^{29}$ there are few that implicate eosinophil-derived cytokines, chemokines, and growth factors in the regulation of cancer growth. Interestingly, as described above, a recent study showed that human eosinophils possessed tumoricidal activity toward a colon cancerous cell lines in culture by releasing TNF. ${ }^{30}$ The tumor-killing effects of eosinophil-derived TNF were the first description of a cytokine, chemokine, or growth factor elaborated by eosinophils implicating a role for these immunomodulators in cancer. The more restricted role of eosinophils and their chemoattractant eotaxin during pubertal ductal morphogenesis is also discussed by Gouon-Evans $\mathrm{V}$ et al., ${ }^{31} \mathrm{~A}$ possible interaction between macrophages and eosinophils in ductal morphogenesis is considered, along with the roles of other chemokines. This role of macrophages in normal development also appears to be subverted by tumors of the mammary gland to promote the escape of the tumor cells from the local environment and enhance their rate of metastasis. These data emphasize the dual role of macrophages in the promotion of epithelial growth in normal and cancer states.

Injection of IL-17E increases the efficiency of chemotherapy and results in eosinophilia. ${ }^{32}$ Eosinophils express IL-17 as determined by immunocytochemistry and Western blot analysis. ${ }^{33}$ Thus, the production of IL-17 from eosinophils may be important in protection of the host against cancers.

Moreover, since eosinophils produce numerous growth factors such as vascular endothelial growth factor (VEGF), as well as a number of other factors that promote angiogenesis, it is possible that eosinophilic inflammation is implicated in tumor neovascularization. ${ }^{34}$ Hypothetically, eosinophil-derived cytokines, chemokines, and growth factors may be involved in enhancing T cell-mediated tumor killing, particularly at the level of the local tissue environment where large numbers of infiltrating eosinophils accumulate and are actively degranulating onto tumor cells. ${ }^{35}$

Taken together, these findings suggest that eosinophils may serve as important components of the immune system, and their cytokines, chemokines, and growth factors may contribute to augment inflammatory responses in allergy and other conditions. Further studies are awaited to understand the involvement of eosinophilderived immunomodulatory factors in the regulation of tumor growth and cancer survival, and the extent to which the release of eosinophil derived factors may be manipulated for therapeutic benefit. Karagiannis et al., ${ }^{14}$ As described by Samoszuk $\mathrm{M}^{17}$ eosinophils may play an important role in the host interaction with the tumor, perhaps by promoting angiogenesis and connective tissue formation adjacent to the cancer. In addition, tumor-related eosinophilia provides some interesting clues into tumor biology, particularly with regard to production of cytokines by the tumor cells. It will be interesting to see how this emerging eosinophil role develops as it seems that eosinophils are a double edged sword that have to be used carefully to harness the benefits and keep the detrimental effects at bay in their role of cancer diagnostics.

It has been hypothecised that eosinophil-derived cytokines, chemokines, and growth factors may be involved in enhancing $\mathrm{T}$ cell-mediated tumor killing, particularly at the level of the local tissue environment where large numbers of infiltrating eosinophils accumulate and are actively degranulating onto tumor cells. Eosinophils may serve as important components of natural immunity, and their cytokines, chemokines, and growth factors may contribute to augment inflammatory responses in allergy, cancer and other conditions.

\section{Acknowledgements}

None.

\section{Conflicts of interest}

Author declares there are no conflicts of interest.

\section{Funding}

None.

\section{References}

1. Broide D, Sriramarao P. Eosinophil trafficking to sites of allergic inflammation. Immunol Rev. 2001;179:163-172.

2. N Taghizadeh, JM Vonk, HM Boezen. Peripheral blood eosinophil counts and risk of colorectal cancer mortality in a large general populationbased cohort study. Journal of Clinical Oncology. 2011;29(Supp1 15):1583-1583

3. Prizment AE, Vierkant RA, Smyrk TC, et al. Tumor eosinophil infiltration and improved survival of colorectal cancer patients: Iowa Women's Health Study. Mod Pathol . 2016;29(5):516-527.

4. Taghizadeh N, Vonk JM, Hospers JJ, et al. Objective allergy markers and risk of cancer mortality and hospitalization in a large population-based cohort. Cancer Causes Control . 2015;26(1):99-109.

5. Gotlib J. World Health Organization-defined eosinophilic disorders: 2015 update on diagnosis, risk stratification, and management. Am J Hematol . 2015;90(11):1077-1089.

6. Cao C, Gu Y, Zhu C, et al. Potential roles of eosinophils in cancer therapy: epidemiological studies, experimental models, and clinical pathology. Recent Pat Anticancer Drug Discov. 2014;9(2):241-248.

7. Need EF, Atashgaran V, Ingman WV, et al. Hormonal regulation of the immune microenvironment in the mammary gland. J Mammary Gland Biol Neoplasia. 2014;19(2):229-239.

8. Karagiannis $\mathrm{SN}$, Josephs $\mathrm{DH}$, Karagiannis $\mathrm{P}$, et al. Recombinant IgE antibodies for passive immunotherapy of solid tumours: from concept towards clinical application. Cancer Immunol Immunother. 2012;61(9):1547-1564.

9. Nicholas JB, Pavlina C, Kathryn LS. Macrophages: Regulators of the Inflammatory Microenvironment during Mammary Gland Development and Breast Cancer. Mediators of Inflammation. 2016;(2016):ID 4549676.

10. Farruggia P, Puccio G, Sala A, et al. AIEOP Italian Association of Pediatric Hematology and Oncology and Hodgkin Lymphoma Working Group. The prognostic value of biological markers in paediatric Hodgkin lymphoma. Eur J Cancer. 2016;52:33-40. 
11. Sabin EA, Kopf MA, Pearce EJ. Schistosoma mansoni egg-induced early IL-4 production is dependent upon IL-5 and eosinophils. $J$ Exp Med. 1996;184(5):1871-1878.

12. Andersen CL, Siersma VD, Hasselbalch HC, et al. Association of the blood eosinophil count with hematological malignancies and mortality. Am J Hematol . 2015;90(3):225-229.

13. Fabre V, Beiting DP, Bliss SK, et al. Eosinophil deficiency compromises parasite survival in chronic nematode infection. $J$ Immunol. 2009;182(3):1577-1583.

14. Gouon-Evans V, Lin EY, Pollard JW. Requirement of macrophages and eosinophils and their cytokines/chemokines for mammary gland development. Breast Cancer Res . 2002;4(4):155-164.

15. Gouon-Evans V, Lin EY, Pollard JW. Requirement of macrophages and eosinophils and their cytokines/chemokines for mammary gland development. Breast Cancer Res. 2002;4(4):155-164.

16. Sakkal S, Miller S, Apostolopoulos V, et al. Eosinophils in Cancer: Favourable or Unfavourable? Curr Med Chem. 2016;23(7):650-666.

17. Samoszuk M. Eosinophils and human cancer. Histol Histopathol. 1997; 12:807-812

18. Farruggia P, Puccio G, Sala A, et al. The prognostic value of biological markers in paediatric Hodgkin lymphoma. Eur J Cancer . 2016;52:33-40.

19. Macdonald D, Gordon AA, Kajitani H, et al. Interleukin-2 treatmentassociated eosinophilia is mediated by interleukin-5 production. $\mathrm{Br} J$ Haematol . 1990;76(2):168-173.

20. Van Haelst Pisani C, Kovach JS, Kita H, et al. Administration of interleukin-2 (IL-2) results in increased plasma concentrations of IL-5 and eosinophilia in patients with cancer. Blood. 1991;78(6):1538-1544.

21. Sosman JA, Bartemes K, Offord KP, et al. Evidence for eosinophil activation in cancer patients receiving recombinant interleukin-4: effects of interleukin-4 alone and following interleukin-2 administration. Clin Cancer Res. 1995;1(8):805-812.

22. Goldsmith MM, Belchis DA, Cresson DH, et al. The importance of the eosinophil in head and neck cancer. Otolaryngol Head Neck Surg. 1992;106(1):27-33

23. Huland E, Huland H. Tumor-associated eosinophilia in interleukin-2treated patients: evidence of toxic eosinophil degranulation on bladder cancer cells. J Cancer Res Clin Oncol. 1992;118(6):463-467.
24. Horiuchi K, Mishima K, Ohsawa M, et al. Prognostic factors for welldifferentiated squamous cell carcinoma in the oral cavity with emphasis on immunohistochemical evaluation. J Surg Oncol. 1993;53(2):92-96.

25. Dorta RG, Landman G, Kowalski LP, et al. Tumour-associated tissue eosinophilia as a prognostic factor in oral squamous cell carcinomas. Histopathology. 2002;41(2):152-157.

26. Lorena SC, Oliveira DT, Dorta RG, et al. Eotaxin expression in oral squamous cell carcinomas with and without tumour associated tissue eosinophilia. Oral Dis. 2003;9(6):279-283.

27. Davoine F, Sim A, Tang C, et al. Eosinophils in human oral squamous carcinoma; role of prostaglandin D2. J Inflamm . 2013;10(1):4.

28. Roth N, Stadler S, Lemann M, et al. Distinct eosinophil cytokine expression patterns in skin diseases - the possible existence of functionally different eosinophil subpopulations. Allergy. 2011;66(11):1477-1486.

29. Gatault S, Legrand F, Delbeke M, et al. Involvement of eosinophils in the anti-tumor response. Cancer Immunol Immunother. 2012;61(9):1527-1534.

30. Legrand F, Driss V, Delbeke M, et al. (2010) Human eosinophils exert TNF-alpha and granzyme A-mediated tumoricidal activity toward colon carcinoma cells. J Immunol. 2010;185(12):7443-7451.

31. Gouon-Evans V, Lin EY, Pollard JW. Requirement of macrophages and eosinophils and their cytokines/chemokines for mammary gland development. Breast Cancer Research. 2002;4:155.

32. Benatar T, Cao MY, Lee Y, et al. Virulizin induces production of IL-17E to enhance antitumor activity by recruitment of eosinophils into tumors. Cancer Immunol Immunother . 2008;57(12):1757-1769.

33. Molet S, Hamid Q, Davoine F, et al. IL-17 is increased in asthmatic airways and induces human bronchial fibroblasts to produce cytokines. J Allergy Clin Immunol. 2001;108(3):43043-43048.

34. Stockmann C, Schadendorf D, Klose R, et al. The impact of the immune system on tumor: angiogenesis and vascular remodeling. Front Oncol . 2014;4:69.

35. Davoine F, Lacy P. Eosinophil cytokines, chemokines, and growth factors: emerging roles in immunity. Front Immunol. 2014;5:570. 